\title{
Morphological segmentation analysis and texture-based support vector machines classification on mice liver fibrosis microscopic images
}

Article

Accepted Version

Wang, Y., Shi, F., Cao, L., Dey, N., Wu, Q., Ashour, A. S., Sherratt, S., Rajinikanth, V. and Wu, L. (2019) Morphological segmentation analysis and texture-based support vector machines classification on mice liver fibrosis microscopic images. Current Bioinformatics, 14 (4). pp. 282-294. ISSN 2212-392X doi:

https://doi.org/10.2174/1574893614666190304125221 Available at https://centaur.reading.ac.uk/75123/

It is advisable to refer to the publisher's version if you intend to cite from the work. See Guidance on citing.

To link to this article DOI:

http://dx.doi.org/10.2174/1574893614666190304125221

Publisher: Bentham Science Publishers

All outputs in CentAUR are protected by Intellectual Property Rights law, including copyright law. Copyright and IPR is retained by the creators or other copyright holders. Terms and conditions for use of this material are defined in the End User Agreement. 


\section{www.reading.ac.uk/centaur}

\section{CentAUR}

Central Archive at the University of Reading

Reading's research outputs online 
Title: $\quad$ Morphological Segmentation Analysis and Texture-based Support Vector Machines Classification on Mice Liver Fibrosis Microscopic Images

Authors:

Yu Wang, College of Information and Engineering, Wenzhou Medical University, Wenzhou, P.R. China

Fuqian Shi, College of Information and Engineering, Wenzhou Medical University, Wenzhou, P.R. China

Luying Cao, College of Information and Engineering, Wenzhou Medical University, Wenzhou, P.R. China

Nilanjan Dey, Department of Information Technology, Techno India College of Technology, West Bengal, India

Qun Wu, Universal Design Institute, Zhejiang Sci-Tech University, Hangzhou, P.R. China

Amira S. Ashour, Department of Electronics and Electrical Communications Engineering, Faculty of Engineering, Tanta University, Tanta, Egypt

R. Simon Sherratt, Department of Biomedical Engineering, the University of Reading, UK

V. Rajinikanth, Department of EIE, St. Joseph's College of Engineering, Chennai, India

Lijun Wu, Institute of Digitized Medicine, Wenzhou Medical University, Wenzhou, P. R. China

Corresponding Author: Fuqian Shi, College of Information and Engineering, Wenzhou Medical University, 325035, Wenzhou, P.R. China; Tel/Fax: +86-577-86689913, +86-57-86699222; E-mail: sfq@wmu.edu.cn

Publication: Current Bioinformatics

Publisher: $\quad$ Bentham Science Publishers

ISSN: $\quad$ ISSN: 2212-392X (Online), ISSN: 1574-8936 (Print)

Volume: $\quad$ not yet assigned

Issue: $\quad$ not yet assigned

Received: $\quad 29^{\text {th }}$ August 2017

Revised: $\quad 29^{\text {th }}$ December 2017

Accepted Date: $\quad 20^{\text {th }}$ January 2018

pp.: $\quad$ not yet assigned

DOI: $\quad$ not yet assigned

\begin{abstract}
:
Background To reduce the intensity of the work of doctors, pre-classification work needs to be issued. In this paper, a novel and related liver microscopic image classification analysis method is proposed. Objective For quantitative analysis, segmentation is carried out to extract the quantitative information of special organisms in the image for further diagnosis, lesion localization, learning and treating anatomical abnormalities and computer-guided surgery. Methods in the current work, entropy based features of microscopic fibrosis mice' liver images were analyzed using fuzzy c-cluster, k-means and watershed algorithms based on distance transformations and gradient. A morphological segmentation based on a local threshold was deployed to determine the fibrosis areas of images. Results the segmented target region using the proposed method achieved high effective microscopy fibrosis images segmenting of mice liver in terms of the running time, dice
\end{abstract}


ratio and precision. The image classification experiments were conducted using Gray Level Co-occurrence Matrix (GLCM). The best classification model derived from the established characteristics was GLCM which performed the highest accuracy of classification using a developed Support Vector Machine (SVM). The training model using 11 features was found to be as accurate when only trained by 8 GLCMs. Conclusion The research illustrated the proposed method is a new feasible research approach for microscopy mice liver image segmentation and classification using intelligent image analysis techniques. It is also reported that the average computational time of the proposed approach was only 2.335 seconds, which outperformed other segmentation algorithms with 0.8125 dice ratio and 0.5253 precision.

Key words: morphological segmentation; top-hat transformation; threshold based Watershed segmentation; texture feature extraction; mice liver fibrosis; microscopic images; support vector machine

\section{INTRODUCTION}

Thresholding is an indispensable image segmentation procedure to outline disjoint regions with connected and closed boundaries. This technique is employed to discriminate the objects of interest, typically in the foreground from the background. Any change in the threshold value can expressively affect the boundary position if the object in the image has steep sides as well as the extracted object overall size. Thus, subsequent object measurements, such as the area are quite sensitive to the threshold value. However, there is no universal approach for the threshold selection with all image types. Several procedures are developed to enable the threshold values determination under different circumstances.

Microscopic digital image processing techniques for feature extraction are carried out to post-process and analyze captured microscopic images. Microscopic image segmentation is a significant process in microscopic image processing. Furthermore, it is considered an important prerequisite for subsequent image identification analysis and classification in several diverse domains, such as medicine, biological research, cancer research, drug testing, and metallurgy. In microscopic mice liver images, the normal liver cells are agglomerated; the pieces can be large or small; and the polygons of cell boundaries are clear, where sometimes cells are visible such as in the small tied gall bladder. Generally, the mice livers' cells have pink cytoplasm, fine granular, round nucleus, centered and stained shallow. In addition, there are also obvious nucleoli and the whole cell distribution is more uniform [1]. In the microscopic cirrhotic or fibrosis mice images, extensive fibrous tissues are re-segmented the original hepatic lobules due to the destruction of the normal hepatic lobule structure including the size of the round or oval-shaped clusters of the liver cell, named pseudolobule. Pseudolobule cells have disordered arrangements and have a certain difference in size of their structure. The overall group are distributed and arranged closely. Thus, there are clusters of regions in the microscopic image, and there are significant differences in the regions compared to other parts of the image.

Typically, microscopic image analysis includes detecting edges of the different objects and counting similar objects as well as calculating the area, perimeter length and other useful measurements of each object. The extracted features of the microscopic image are used for further quantitative analysis measurements and feature identification of normal and abnormal cases in the dataset. This can be used widely in testing for example the blood cells and thyroid tissues for significant clinical and biological interpretable features [2][3][4]. Rakotomamonjy et al. extracted scattering features for lung cancer detection in fibered confocal fluorescence microscopy images [5]. Texton based image features [6] is another extracted feature that can be used for further image processing. Nellros et al. addressed particle joins features from images and performed better effectiveness than traditional pellet diameter measurements [7]. Genetic Algorithm (GA) and Particle Swarm Optimization (PSO) in the context of light microscopy imaging systems have been also introduced [8].

Recently, mathematical morphological analysis has been introduced to improve the microscopic image segmentation. Morphology has a complete mathematical basis providing a significant foundation for morphological characteristics analysis and system design of image analysis and image processing. Hence, morphological filters are especially prominent is the realization of the morphological analysis and parallel processing algorithms that greatly improve the speed of image processing and image analysis. Chun and Kong evaluated the conventional gradient operators in terms of autofocusing resolution metric for thermal infrared (IR) images [9]. Zia et al. introduced feature/edge-preserving noise removal techniques based on the morphological gradient [10]. Li et al. introduced an Adaptive Morphological Gradient Lifting Wavelet (AMGLW) algorithm for detecting bearing defects better than the lifting wavelet and linear wavelet transform [11]. Hereafter, Khakipour et al. developed a morphological gradient wavelet method for real-time signal processing [12]. Furthermore, other improved technical relatives to morphology were developed, such as the tensorial morphological gradient approach [13], sparse feature matrix decomposition and morphological filtering [15], morphological center operator [16], which are widely applied in color eye images [17], bacteriophages automatic detection [18], and Ensis directus [19]. Oschatz et al. proposed a morphological analysis method for transmitting electron microscopy images combined with physisorption and Raman spectroscopy [20]. Moreover, Kayasandik and Labate improved detection of soma location and morphology in fluorescence microscopy images of neurons [21]. Furthermore, Non-Alcoholic Steatohepatitis (NASH) has 
been used as a common liver disorder for detecting morphological changes [22]. López-Mir et al. proposed liver segmentation of magnetic resonance images [23].

Watershed is a transformation definition on grayscale images that is metaphorical to a geological watershed, or drainage divide, which separates the adjacent drainage catchment basins [23]. It is also widely used in medical image analysis, such as human skin region segmentation [24]. The Watershed algorithm does have some disadvantages, such as over segmenting on the liver muscle area and density measures [25] [26]. It has been reported that there is some improvement that do need to be found while used to analyze liver images, such as corridor reduction [27].

Consequently, automated image segmentation methods are useful when identifying different regions, and for follow-up tasks based on image classification and lesion area identification analysis for improved diagnosis accuracy [28] [29] [30]. However, mice liver tissues are different from other tissues. The pseudolobule can be the main diagnostic feature for liver fibrosis, thus identification was the main goal of the current work. The difference between the tissue region and the normal tissue region of the lesion (sample/specimen) is the texture feature of the region [31]. The goal of the image segmentation method using image texture feature analysis is based on subdividing the image into different regions with different texture features. For different texture features, different structural elements of the original image reconstruction process based on the mathematical morphology method was used as our image segmentation algorithm. Establishing a strict mathematical theory is the motivation of the current work since the emerging discipline with quantitative geometric characteristics and structural properties description and analysis is the main context of this research. Our method is also a new method that can be applied in the field of image processing and pattern recognition. Since previously, several studies depicted that some improved watershed segmentation algorithms were feasible for segmenting colored image, magnetic resonance brain images, tissues and cells. In the current work, liver fibrosis images segmentation of mice liver images was performed based on the local threshold and compared to other watershed segmenting operations. The watershed segmentation, fuzzy C-means clustering (FCM) and k-mean methods are conducted in the current work for comparative study [32].

It is inevitable that a certain degree of missed diagnosis and misdiagnosis is inevitable, and the application of Computer-Aided Diagnosis (CAD) can be used to alleviate problem. At present, most of the field of liver assistance diagnosis uses B-mode ultrasound or Computer Tomography (CT), and the scanning data can be the imaging basis for their diagnosis. Li et al. used the scanning data obtained during liver CT image based on texture feature extraction using a variety of methods to train the artificial neural network classifier, in normal and tumor tissue, cyst and other lesions, three diagnostic sub problems of hepatocellular carcinoma and liver hemangioma, the diagnostic accuracy rate reached $0.9797,0.9851$ and 0.9753 respectively [33]. Gray level co-occurrence matrix, Lloyd's spectral feature measure and other methods to classify the liver ultrasound data of normal tissue, cyst, benign and malignant cancer, obtained satisfactory results [34].

In this paper, the application of texture feature extraction using Gray Level Co-occurrence Matrix (GLCM) and a Support Vector Machine (SVM) for image processing of the mice liver micro image in order to extract texture features of microscopic images is addressed. This work has more accurately distinguished normal tissue image and image characteristics of liver cirrhosis and has laid the foundation for the realization of the next automatic CAD systems.

Based on the local threshold segmentation and morphological processing, the current work divided the target region of the original mice images using fuzzy $\mathrm{C}$-means and the $\mathrm{K}$-means clustering segmentation methods compared to using the watershed division using the gradient and the distance. The features using the different image segmentation algorithms were analyzed according to the experimental results. The proposed method is applied to solve the lesion area of the liver microsystem image.

The organization of the remaining sections is as follows. Section 2 presents the methods for image segmenting and the experimental process. Section 3 addresses the results and comparative analysis. Section 4 involves the conclusions of the present study.

\section{METHODS AND MATERIALS}

\subsection{Mathematical morphology}

Set theory has been used to describe the mathematical morphology operations in the digital image processing procedures. Consider an image containing a Region of Interest (ROI) marked by $(A, B, C, \ldots)$. The single element represents a pixel in the 2D image of the position coordinated as $z=\left(z_{1}, z_{2}\right)$, where $z \in Z^{2}$. The basic idea of morphology based image processing is to use the structural elements to collect the image information. When the structural elements continue to move in the image, the relationship between the various parts of the image can be observed. In order to understand the various parts of the image structure, structural elements can directly carry out as shape, size, grayscale and chrominance information to detect the structural features of the target images. Manipulating and analyzing the image by a series of structural transformations between different shapes of structural elements is adopted. Commonly, it is a nonlinear filtering method including morphology and difference (expansion and corrosion) as well as close and open operation [23]. 


\subsubsection{Corrosion and expansion}

Corrosion and expansion are two basic and important morphological operations having the following theoretical description basis. The corrosion on $A$ using $S$ is denoted as $A \Theta S$, where $A$ and the moving structure element $S$ are in the plane $Z^{2}$, which can be formalized as follows:

$$
A \Theta S=\left\{Z \mid(S)_{z} \subseteq A\right\}
$$

All points $Z$ is $A \Theta S$ if $S$ is fully involved in $A$, while the origin of $S$ moves to $Z$. Moreover, the expansion for any set $A$ and $S$ in $Z^{2}, \phi$ is empty set, expansion on $A$ using $S$ is denoted as $A \oplus S$ and can be formalized as:

$$
A \oplus S=\left\{Z \mid\left(S^{*}\right)_{Z} \cap A \neq 0\right\}
$$

where, originally, the structure elements $S$ are located in the origin of the image moving in the entire plane $Z^{2}$, when its own origin shift to $Z$, i.e., $S^{*}$ has at least 1 pixel overlap with $A$. All such points $Z$ is the expansion image of $A$ using $S$.

\subsubsection{Open and close}

Open and close operations are compounded by corrosion and expansion. In general, the open operation smooths the image contour and disconnects the narrow connection and removes burrs. Closed operations can also make the contour smooth, but contrary to the open operation, it is usually able to narrow the gap and fill the small holes. Consequently, the open operation on image $A$ using structure element $S$ can be formalized as:

$$
A \cdot S=(A \oplus S) \Theta S
$$

In addition, the close operation on image $A$ using structure element $S$ can be formalized as:

$$
(A \cdot S)^{c}=A^{c} \circ S^{c}
$$

where,

$$
A \circ S=(A \Theta S) \oplus S
$$

\subsection{Local threshold based morphological segmenting}

The basic idea of threshold segmentation is to determine a threshold and then compare the gray value of each pixel and the threshold value. Afterwards, according to the results, the pixels are divided into two categories, namely the foreground and background. The threshold segmentation method is usually based on the experimental method including histogram threshold method, iterative threshold method, the minimum mean square error method and the maximum between class variance method. In the application of local threshold method, the intra-class variance or the variance within the class was adopted in the current work.

\subsubsection{Maximum interclass variance method}

In image segmentation, the segmentation threshold should be selected between the average gray of the image, and the average gray background foreground regions of the difference based on the difference in the regional variance. Otsu's put forward the maximum variance method in 1978. Based on the principle of least squares method, the algorithm is simple and stable. Suppose that the number of pixels with $i \in[0, L-1]$ grayscale is $n_{i}$, where the total number of pixels is:

$$
N=\sum_{i=0}^{L-1} n_{i}
$$

The probability of each grayscale is expressed by:

$$
p_{i}=\frac{n_{i}}{N}
$$

For $p_{i}$, the following expression is valid:

$$
\sum_{i=0}^{L-1} p_{i}=1
$$

The divided pixels in the image using threshold $T$ as two classes $C_{0}$ and $C_{1}$, where $C_{0}$ is consistent of the pixels whose grayscale is in $[0, T-1]$ and $C_{1}$ is in $[T, L-1]$, then the probability of zones $C_{0}$ and $C_{1}$ are given by:

$$
\begin{gathered}
p_{0}=\sum_{i=0}^{T-1} p_{i} \\
p_{1}=\sum_{i=T}^{L-1} p_{i}=1-p_{0}
\end{gathered}
$$

The average grayscale of $C_{0}$ and $C_{1}$ are given by: 


$$
\begin{aligned}
& \mu_{0}=\frac{1}{p_{0}} \sum_{i=0}^{T-1} i p_{i}=\frac{\mu(T)}{p_{0}} \\
& \mu_{1}=\frac{1}{p_{1}} \sum_{i=T}^{L-1} i p_{i}=\frac{\mu-\mu(T)}{1-p_{0}}
\end{aligned}
$$

where, $\mu$ is average grayscale of entire image calculated by:

$$
\mu=\sum_{i=0}^{L-1} i p_{i}=\sum_{i=0}^{T-1} i p_{i}+\sum_{i=T}^{L-1} i p_{i}=p_{0} \mu_{0}+p_{1} \mu_{1}
$$

The variance between these two zones is given by:

$$
\sigma_{B}^{2}=p_{0}\left(\mu_{0}-\mu\right)^{2}+p_{1}\left(\mu_{1}-\mu\right)^{2}=p_{0} p_{1}\left(\mu_{0}-\mu_{1}\right)^{2}
$$

Let $T$ be ergodic values in $[0, L-1]$ that maximizes $\sigma_{B}^{2}$. The proposed method does not need to set any other parameters; it is a method of automatic threshold selection for better results. It can be applied not only to the single threshold selection with two regions, but also to the multi-region multi-threshold selection.

\subsubsection{Top-hat transformation}

One of the most important applications of the gray morphology using top hat transformation is to provide ideal effect in solving the problem of non-uniform illumination. The top hat transform $h$ is defined as the opening difference of an image $f$ and its structure elements $s$, which is expressed by:

$$
h=f-(f \circ s)
$$

It is difficult to obtain a satisfactory result by thresholding the image directly under the condition that the image is not uniform, while top hat transformation can:

Step 1: use an adaptive optimal threshold method for segmentation;

Step 2: determine the appropriate structural element and image to perform the open operation to eliminate the peak value in adaptive threshold results, and,

Step 3: produce an image open operation result.

The algorithm of the proposed local threshold based morphological segmentation of the microscopic mice liver images is presented in Algorithm 1.

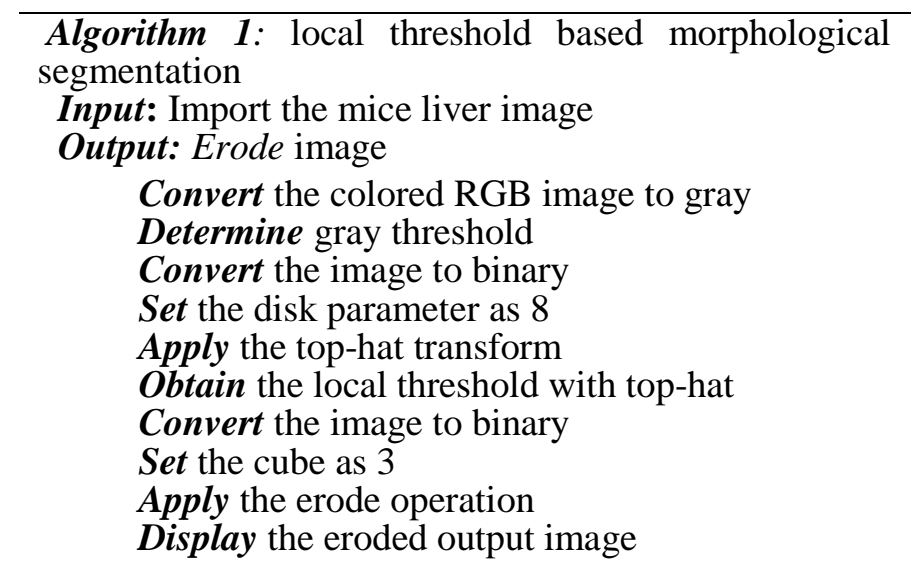

The flowchart of Local threshold based morphological segmenting with Top hat transformation is illustrated in Fig. 1 


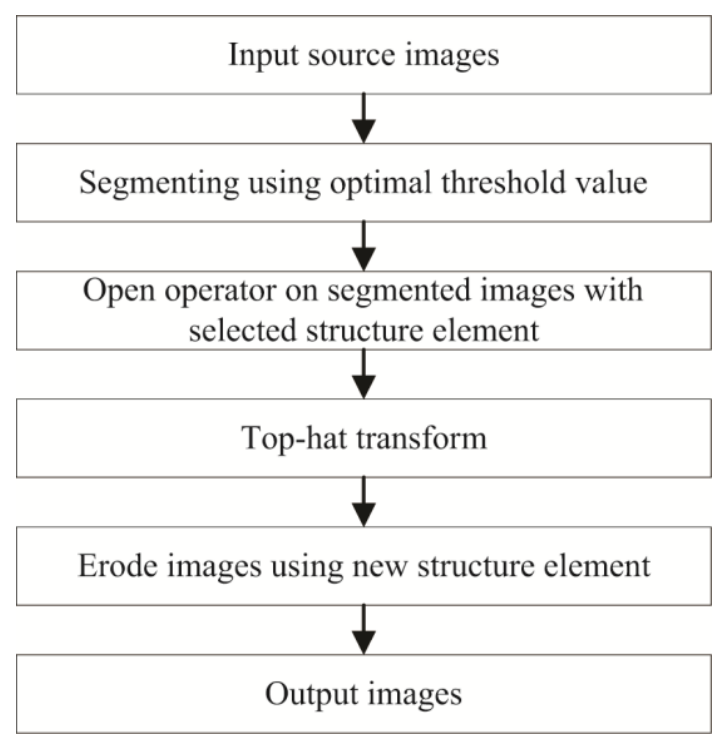

Figure 1. The flowchart of morphological segmenting with Top hat transformation

\subsection{Watershed segmentation}

The basic idea of the watershed algorithm is to assume a hole in the minimum value of each area; and then let the water rise at a uniform rate through the hole to flood the entire terrain. Rising the catchment basin builds a dam to prevent the polymerization. The water reaches the top of each dam on the waterline. The boundaries of these dams correspond to the dividing lines of the watershed is acquired.

\subsubsection{Basin construction of watershed}

Expansion method can be used to separate the two classes' points' catchment basins. Fig. 2(a) is the $(n-1)^{\text {th }}$ basin and Fig. 2(b) shows the flooding result at step $n$ and Fig. 2c) shows water flooding between basins and the dam is needed for preventing its occurrence. Let $M_{1}$ and $M_{2}$ be sets of the minimum points in these two areas, then, the minimums are $C_{n-1}\left(M_{1}\right)$ and $C_{n-1}\left(M_{2}\right)$ in step $n-1$ relative to the gray area in Fig. 2(c). Let $C[n-1]=C_{n-1}\left(M_{1}\right) \cup C_{n-1}\left(M_{2}\right)$ and two connective components in Fig. 2(a), while one in Fig. 2(c) illustrates that expansion needs to be limited by a connective component.

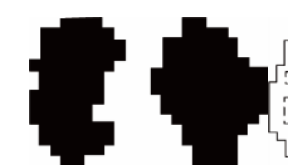

(a)

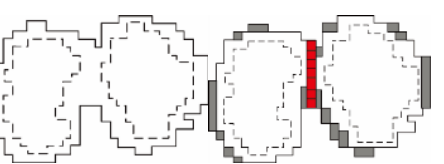

(c)

Figure 2. watershed with flooding algorithm

\subsubsection{Watershed based segmenting algorithm}

With $M_{1}, M_{2}, \ldots, M_{R}$ sets of the minimum points of an image $g(x, y)$, and $C\left(M_{i}\right)$ be the coordinates of points in basin relative to $M_{i}, T[n]=\{(s, t)\}$ satisfying $g(s, t)<n$; following the waterline increasing from $n=\min \{g(x, y)\}$ to $n=\max \{g(x, y)\}+1$, for step $n$ of the flooding process, the algorithm outputs the number of points in the submerged depth. Conceptually, the coordinates are assumed to be black under the plane, i.e. all $T[n]$ subjected to $g(x, y)<n$, and all other coordinates are marked white. Then, at any step $n$, look down the plane to acquire the binary image, the black points correspond to the point below the plane. Let $C_{n}\left(M_{i}\right)$ be the coordinates set relatives to $M_{i}$ in basin, thus:

$$
C_{n}\left(M_{i}\right)=C\left(M_{i}\right) \cap T[n]
$$

Then in $(x, y), C_{n}\left(M_{i}\right)=1$ or 0 . Noted that:

$$
C[n]=\bigcup_{i=1}^{R} C_{n}\left(M_{i}\right)
$$


In addition,

$$
C[\max \{g(x, y)\}+1]=\bigcup_{i=1}^{R} C\left(M_{i}\right)
$$

The elements in $C_{n}\left(M_{i}\right)$ and $T[n]$ are not be replaced and increased while $n$ is increased. Hence, $C[n-1] \subset C[n]$, thus, $C[n-1] \subset T[n]$. The algorithm is used to find the dividing line $C[\min \{g(x, y)\}+1]=T[\min \{g(x, y)\}+1]$ and calculate $C[n]$. Let $Q$ be set of all connective components $q$ of $T[n]$. For each $q \in Q[n]$, there are three conditions:

1) $q \bigcap C[n-1]$ is an empty set.

2) $q \bigcap C[n-1]$ involves a connective component of $C[n-1]$.

3) $q \cap C[n-1]$ involves one more connective components of $C[n-1]$.

New minimum point goes to the system, $q$ is added to $C[n-1]$ by condition (2) when $q$ is in some local minimum catchment basin. Condition (3) occurs when all or part of the ridge line separates the two or more of the catchment basin leading to further submerged water catchment basin polymerization. Therefore, a dam in $q$ (if it involves more than two of the catchment basin, a dam is required to be built between the water overflow drainage basin). The algorithm of the gradient based watershed segmentation is presented in Algorithm 2.

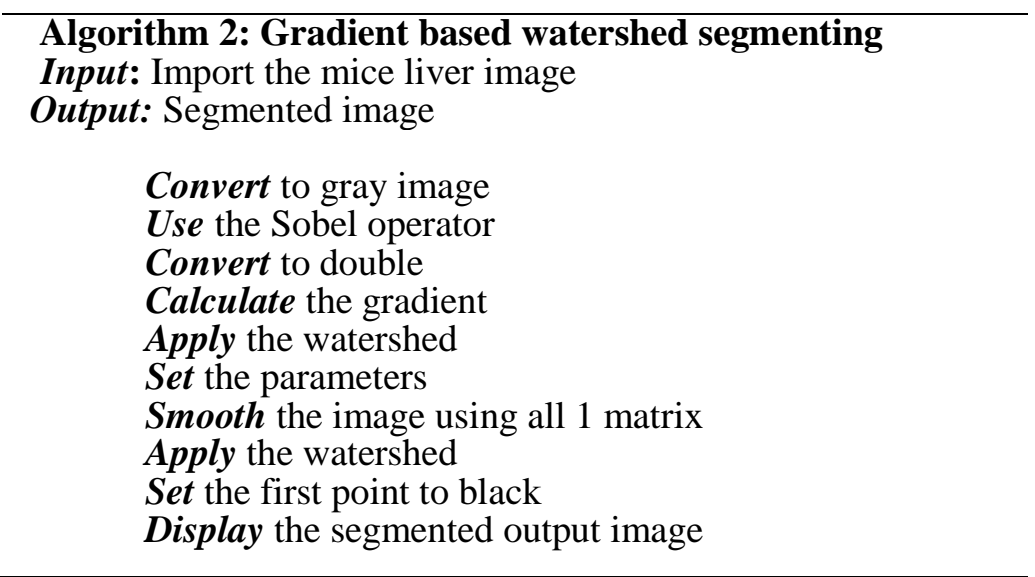

\subsection{Entropy based GLCM Features for SVM Classification}

\subsubsection{Entropy calculation}

Using the concept of information entropy in information theory, the entropy (average self-information) of any discrete source is obtained. Self-information is a random variable. It refers to the amount of information that a certain source sends out a message. The amount of information in a piece of information has a direct bearing on its uncertainty. The messages sent are different, and contains different amounts of information. The self-information of any message cannot represent the average self-information contained in the source. The significance of the information entropy of an image represents the aggregation characteristics of the gray distribution of an image. The image entropy is expressed as the bit mean of the image gray level set, the unit bit / pixel, and the average information of the image source are also described. As the basic of GLCM, the entropy was as addressed; since the single grey point is independent, then the image gray distribution $P=\left\{P_{0}, P_{1}, \cdots P_{K}\right\}$, in which $P_{k}$ represents the probability of the gray value of image pixel is $k$ in $F(i, j)$, thus the entropy is given by: $E=-\sum_{i=0}^{L-1} P_{i} \log _{2} P_{i}$. where, $L$ was the total grey levels of the image.

\subsubsection{Texture based features}

Each image has its own features that distinguish it from other types of images, such as brightness, edges and textures. Some of these features need to be obtained by transformation or processing, such as histograms and principal components. The feature extraction methods include statistical methods, spectral features and structural features. The corresponding methods include gray level co-occurrence matrix method and spectrum based analysis method. 


\section{1) Gray Level co-occurrence matrix method}

The texture is formed by the gray distribution in the alternately changed space position, there will be a gray relation between two pixels, and therefore it has a certain relationship in spatial location in the image, known as the spatial correlation of image intensity. Describing texture using gray level spatial correlation is the ideological foundation of the GLCM. The GLCM is a classical and commonly used method of texture statistical analysis. Let $P_{\delta}$ be GLCM, gray level be $L$, then $P_{\delta}$ is a $L \times L$ matrix and element $P_{\delta}(i, j)$, $(i, j=0,1,2 \ldots, L-1)$ is defined as probability of space relation $\delta=\left(D_{x}, D_{y}\right)$ and frequency of pixels $i$ and $j$. Space position relations have four types in vertical, horizon and $\pm 45^{\circ}, P_{\delta}$ can be generated using:

$$
P_{\delta}=\left[\begin{array}{cccccc}
P_{\delta}(0,0) & P_{\delta}(0,1) & \ldots & P_{\delta}(0, j) & \ldots & P_{\delta}(0, L-1) \\
P_{\delta}(1,0) & P_{\delta}(1,1) & \ldots & P_{\delta}(1, j) & \ldots & P_{\delta}(1, L-1) \\
\ldots & \ldots & \ldots & \ldots & \ldots & \ldots \\
P_{\delta}(i, 0) & P_{\delta}(i, 1) & \ldots & P_{\delta}(i, j) & \ldots & P_{\delta}(i, L-1) \\
\ldots & \ldots & \ldots & \ldots & \ldots & \ldots \\
P_{\delta}(L-1,0) & P_{\delta}(L-1,1) & \ldots & P_{\delta}(L-1, j) & \ldots & P_{\delta}(L-1, L-1)
\end{array}\right]
$$

GLCM describes the two-order statistical feature of image texture and is suitable for many species; the advantages of GLCM are easily description on the spatial relationship between pixels in color, and it is invariant of gray level transformation. Commonly used feature parameters for GLCM are texture, two-rank angle, contrast, correlation, entropy, etc.

\section{2) Gabor wavelet transform}

Suppose that $f(x, y)$ of size $M \times N$, the 2-dimensional discrete Gabor transform is:

$$
I_{p, q}(x, y)=\sum_{s} \sum_{t} f(x-s, y-t) \varphi_{p, q}^{*}(s, t)
$$

where, $s$ and $t$ are variables of size of filter mask, $x$ and $y$ are coordinates of pixels; $p$ and $q$ represent the scale and direction of the wavelet, $p=0, \ldots, P-1$, and $q=0, \ldots, Q-1 . P$ and $Q$ represent all scales and directions of wavelet. $\varphi_{\mathrm{p}, q}{ }^{*}$ denotes complex conjugate of $\varphi_{\mathrm{p}, q}$ and $\varphi_{\mathrm{p}, q}$ is a wavelet transform function of Gabor, that can be presented as:

$$
\varphi_{\mathrm{p}, q}(x, y)=a^{-p} \varphi\left(x^{\prime}, y^{\prime}\right)
$$

where, $a>1$ as factor of scale, and:

$$
x^{\prime}=a^{-p}(x \cos \theta+y \sin \theta), \quad y^{\prime}=a^{-p}(-x \sin \theta+y \cos \theta)
$$

where, $\theta=\mathrm{q} \pi / Q$. If we use the Gabor function as mother wavelet, then it can be presented as:

$$
\varphi_{\mathrm{p}, q}=\left(\frac{1}{2 \pi \sigma_{x} \sigma_{y}}\right) \exp \left(-\frac{1}{2}\left(\frac{x^{2}}{\sigma_{x}^{2}}+\frac{y^{2}}{\sigma_{y}^{2}}\right)\right) \exp (2 \pi j w x)
$$

where, $w$ presents bandwidth of Gabor, $\sigma_{x}$ and $\sigma_{v}$ present constants of Gaussian envelope following directions $x$ and $y$. Therefore, according to the results of two-dimensional Gabor wavelet transform, the image means and standard variance, contrast and entropy can be calculated as texture features.

\subsubsection{Support Vector Machine}

\section{1) Recognition methods using SVM}

SVM is a relatively new generation of learning algorithm whose development was based on statistical learning theory. For linearly separable training samples $(x, y)$, a classifier surface can be separated without error. The greater the spacing between categories, the better the generalization. The largest classification surface is the optimal classification surface. By applying the two-programming method, we can find that the optimal classification function is:

$$
\mathrm{f}(x)=\operatorname{sgn}\left(\sum_{i=1}^{n} T_{i} y_{i}\left(x_{i} \cdot x\right)+b^{*}\right)
$$

where, $T_{i}$ is the Lagrange operator and $b^{*}$ is threshold of classification. For nonlinear un-separable, nonnegative relax term $\alpha_{i}$ and $C$ can be inducted to get optimal classification (same as Eqn. (5).) For nonlinear problems, it can be transformed into a linear problem in high-dimensional space by kernel functions, and then the optimal classification plane is obtained in the transformation space, Eqn. (24) can be transformed as:

$$
\mathrm{f}(x)=\operatorname{sgn}\left(\sum_{i=1}^{n} T_{i} y_{i} K\left(\mathrm{x}_{i}, x_{j}\right)+b^{*}\right)
$$


The kernel function may include:

$$
\begin{gathered}
\text { Linear }-K(x, y)=x \cdot y \\
\text { Polynomial }-K(x, y)=(x \cdot y+1)^{d}, d=1,2, \ldots \text { (27) } \\
\text { Radial basis function }-K(x, y)=\exp \left(-r\|\mathrm{x}-\mathrm{y}\|^{2}\right) \\
\text { Sigmoid }-K(x, y)=\tanh (b(x \cdot y)-c)
\end{gathered}
$$

\section{2) Parameters optimization of SVM}

When using SVM to do the classification prediction, it is needed to adjust the related parameters (primarily the penalty parameter $C$ and the kernel function parameter $g$ ), and the ideal parameters need to be selected for obtaining good prediction accuracy. This study uses cross validation (K-Cross Validation (K-CV)) theory, that is, the original data is divided into $K$ groups, and each data subset respectively a validation set, the $K-1$ group and the rest of the subset of data as the training set results in $K$ models. Using $K$ models, the average number of classification accuracy of the final set is used as the performance index of the classifier under this $\mathrm{K}-\mathrm{CV}$. This method can effectively avoid the occurrence of over learning and under learning, and finally achieve better accuracy for the prediction of the test set.

\section{RESULTS AND DISCUSSION}

\subsection{Experiment Design}

Microscopic images were obtained from experimental liver fibrosis model of 50 bread male Swiss albino mice from the Theodore Bilharz Research Institute, Giza, Egypt. A magnification of 3400 using a Leica microscope MD500 with software version 1.8.0 was used. The original acquired fibrosis liver microscopic images are illustrated in Fig. 3(a)-(h). Gray images are shown in Fig. 4(a)-(h).

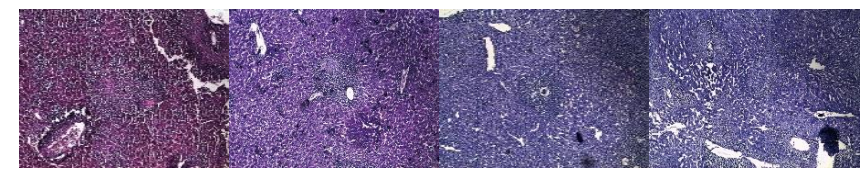

(a)

(b)

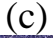

(d)

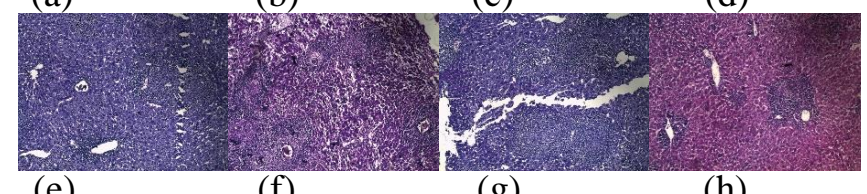

(e)

(f)

(g)

(h)

Figure 3. Original microscopic images of fibrosis mice liver
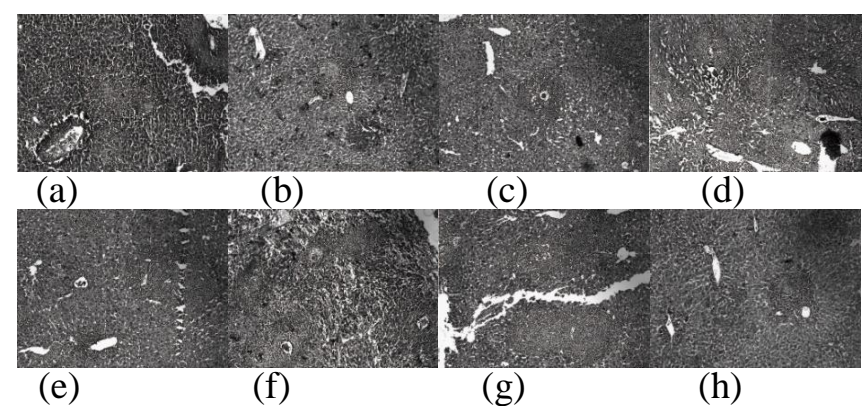

Figure 4. Gray transform of fibrosis mice liver microscopic images 
The binary images of the dataset using Otus's threshold based segmenting are shown in Fig. 5.

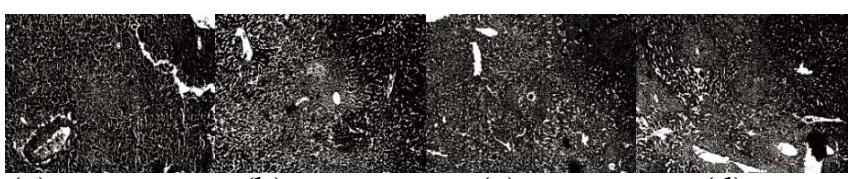

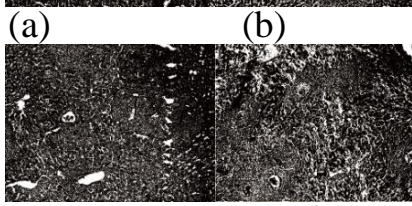

(e)

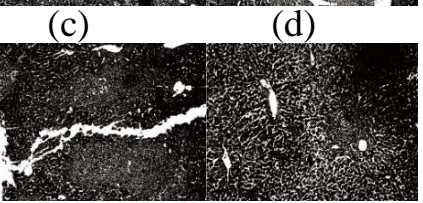

(g)

(h)

Figure 5. Otsu's threshold segmenting based binary images

In the present work, eight images are processed based on the local threshold segmentation and watershed algorithms. For comparative study, the sixth image (f) is used to illustrate the differences between the proposed method and the other segmentation algorithms. In Fig. 5, there are four cell clusters in the image, and the cell distribution is dense. Table 1 listed the local threshold values using Otsu segmentation.

Table 1 Eight fibrosis mice liver microscopic images

\begin{tabular}{|c|c|}
\hline Image_ID & $\begin{array}{c}\text { Local Threshold using Otsu } \\
\text { segmentation }\end{array}$ \\
\hline a & 0.4863 \\
\hline b & 0.4784 \\
\hline c & 0.4627 \\
\hline d & 0.5176 \\
\hline e & 0.4549 \\
\hline f & 0.4627 \\
\hline g & 0.5098 \\
\hline h & 0.4275 \\
\hline Average & 0.4750 \\
\hline
\end{tabular}

The results are shown in Fig. 6 and Fig. 7 using the fuzzy C-clustering and K- mean clustering methods to segment the target area.

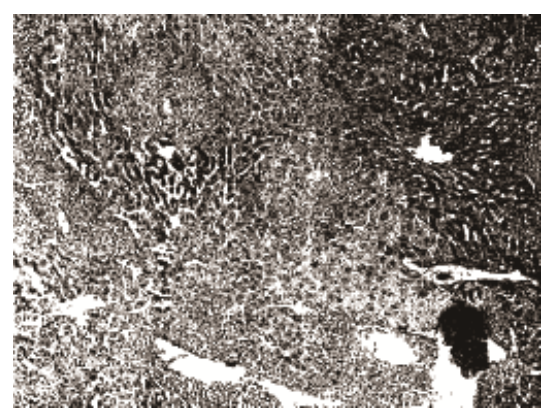

Figure $6 \mathrm{FCM}$ by class number $=3$

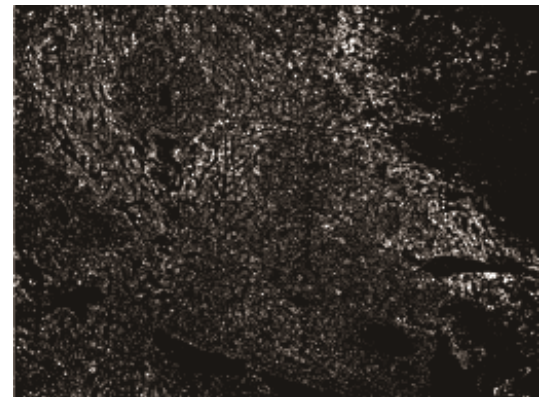

Figure $7 \mathrm{k}$-mean clustering with class number $=3$ 
Fuzzy C clustering segmentation has the advantages of unsupervised, easy to implement, and being adaptive to the uncertainty in an image. However, as can be seen in Fig. 6, the target region and background difference are not obvious, in many parts blending occurs. Thus, for the microscopic image of the unknown complex structure, it is difficult to be clearly divided into several categories. Moreover, the FCM results in clustering the initial value of the influence of the mixed target distribution. The resulting confusion means it is hard to obtain the ideal results as illustrated in Fig. 5. The experimental results of image are also very easy to find. Due to the existence of similar gray values in the image object region and other regions, the target and background areas' clustering results cannot be distinguished and still mixed together with clustering errors.

In Fig. 7, the K-means clustering provided better results than the FCM. However, the target area is noise polluted, and the segmentation is flawed, resulting in difficulty for identification. The result of the K-means algorithm corresponds to the hard segmentation, and the result of the FCM algorithm corresponds to the soft segmentation. In the experiment of K-means algorithm, the number of clusters is set to 3 ; the cluster region is set as the pathological region, the background and the normal tissue region. The method is essentially a hard segmentation method; the clustering error caused by the combination between regions can be avoided. Furthermore, the morphological top-hat processing on the obtained images is illustrated in Fig. 8. In addition, the results are show in Fig. 9 showing the local threshold segmentation output.

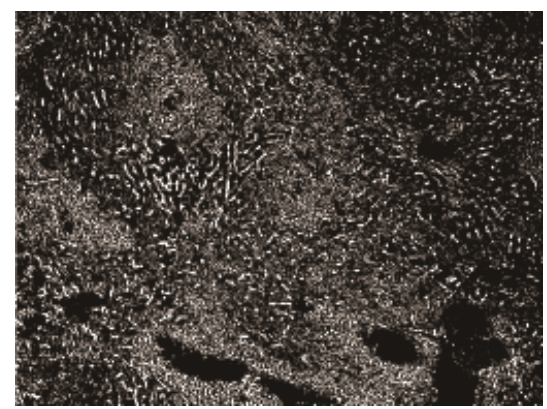

Figure 8 Top-hat transform with local threshold

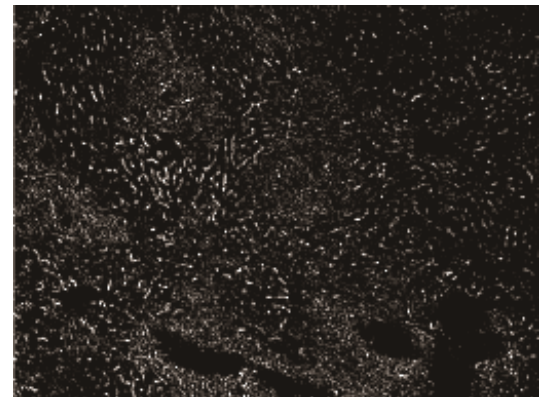

Figure 9 Morphological erode result

Watershed segmentation using the distance transform and gradient are shown in Fig. 10 and Fig. 11.

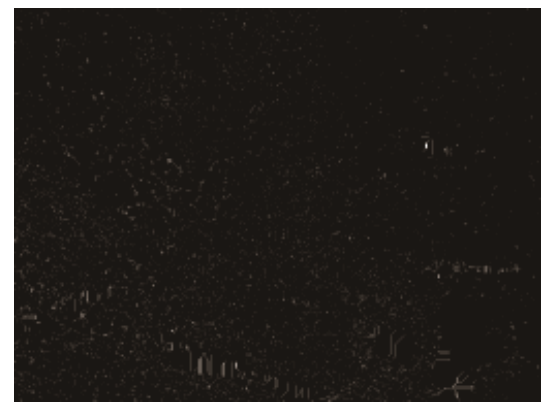

Figure 10 Watershed segmenting with distance transform 


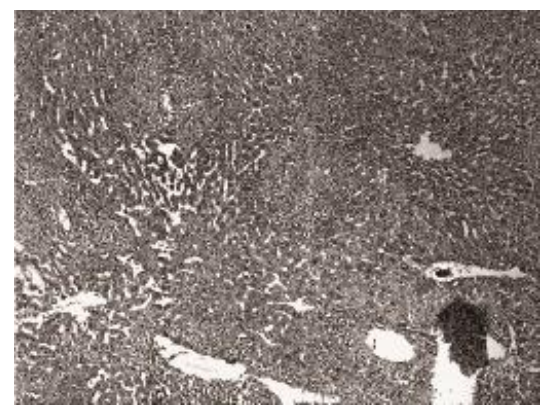

Figure 11 watershed segmenting with gradient

The comparative study of the experimental results in Fig. 10 and Fig. 11 of the three image segmentation methods indicate that morphological processing is better than that of watershed segmentation and clustering segmentation. As shown in Fig. 10 and Fig. 11 using the Sobel operator and structure element is identity matrix of $3 \times 3$. There is a serious over- segmentation problem in the watershed segmentation. The main application of watershed segmentation method is to extract the objects from the background. The gradient values of the regions with small changes in the gray level are also small, so the watershed segmentation method is more significant compared to the gradient of the image. In the current work, the gray scale difference of the mouse liver micrograph is not obvious, so the use of gradient watershed segmentation cannot get the desired results.

Generally, the obtained results using local threshold based morphological processing are shown in Fig. 8 and Fig. 9. Compared with the FCM, K-mean clustering method and the watershed segmentation results, it is depicted that the regional assembly and the pixel aggregation characteristics of high density can be retained. In addition, the binary images and their results using morphology Top hat transformation obtained by single Otsu's method, the region has a good background split out, and did not affect the target area. This is mainly where the original image is non-uniform in the imaging light gray image overall caused by uneven, resulting in a single threshold method and cannot achieve the desired results. Moreover, further results of morphological processing to the image are shown in Fig. 8, the structure elements are selected to "cube" model and parameter is 3. By further processing, the complexity of the image is simplified and the ideal result is achieved under the condition of preserving the feature of the lesion area.

This study acquired 16 normal mice liver microscopic images and 16 cirrhosis images. The normal image is labeled as a positive sample, and the liver cirrhosis image is labeled as a negative sample. These images have been consulted by the pathologist and confirmed from the same mice. Fig. 3 shows part of those 32 images. Mice liver cirrhosis has the key features of pseudolobuli, and with the emergence of tissue fibrosis, the characterization of the image pixels to pixels can be found as uneven distribution and large amplitude of changes. Therefore, in the selection of feature dimensions, the emphasis is placed on the detection of spatial positions and directions. We use gray level co-occurrence matrix method and selected 8 feature parameters for all images. Similarly, 3 characteristic parameters are selected by Gabor wavelet transform. The characteristic dimensions of the selection are shown in Table 2.

Table 2 Features selection for GLCM and Gabor wavelet

\begin{tabular}{|c|c|}
\hline GLCM & $\begin{array}{c}\text { Gabor Wavelet } \\
\text { Transform }\end{array}$ \\
\hline 2-Rank Moment & Binarization Uniformity \\
\hline Inertia Moment & Binarization Variance \\
\hline Entropy & Binarization Entropy \\
\hline Correlation & \\
\hline Uniformity & \\
\hline Variance & \\
\hline Non-Similarity & \\
\hline $\begin{array}{c}\text { Inverse Difference } \\
\text { Moment }\end{array}$ \\
\hline
\end{tabular}




\subsection{Classification result using SVM}

In this study, all the feature dimensions are combined to form a $32 \times 11$ data set, and then 10 images are randomly extracted from two sets of images, and a total of 20 images are formed. Then, the test set with the remaining 12 image data was formed. Then, the original data is normalized. Finally, we established a SVM model, first using the training set to train the model, and then the test set prediction, the kernel function used in the model was the polynomial kernel function. In parameter optimization, 2 -fold cross validation, 5 -fold cross validation, and test set optimization methods are adopted respectively, and the results are shown in Table 3.

Table 3 The parameters of the best classification accuracy in the experiment of image classification

\begin{tabular}{|l|l|l|l|l|}
\hline Features & Methods & Penalty function -c & Kernel-g & Accuracy (\%) \\
\hline \multirow{8}{*}{8} & $\begin{array}{l}\text { 2-fold cross } \\
\text { validation. }\end{array}$ & 2.2974 & 4.0000 & 60.0 \\
\cline { 2 - 5 } & $\begin{array}{l}\text { 5-fold cross } \\
\text { validation. }\end{array}$ & 0.2500 & 1.8661 & 60.0 \\
\cline { 2 - 5 } & $\begin{array}{l}\text { test set } \\
\text { optimization }\end{array}$ & 1.2311 & 1.8661 & 66.7 \\
\hline \multirow{3}{*}{11} & $\begin{array}{l}\text { 2-fold cross } \\
\text { validation. }\end{array}$ & 0.2500 & 2.4623 & 90.0 \\
\cline { 2 - 5 } & $\begin{array}{l}\text { 5-fold cross } \\
\text { validation. }\end{array}$ & 0.2500 & 0.2500 & 85.0 \\
\cline { 2 - 5 } & $\begin{array}{l}\text { test set } \\
\text { optimization }\end{array}$ & 0.2500 & 3.2490 & 91.7 \\
\hline $\begin{array}{l}\text { 2-fold cross } \\
\text { validation. }\end{array}$ & 0.2500 & 0.2500 & 80.0 \\
\hline $\begin{array}{l}\text { 5-fold cross } \\
\text { validation. }\end{array}$ & 0.2500 & 0.2500 & 80.0 \\
\cline { 2 - 5 } & $\begin{array}{l}\text { test set } \\
\text { optimization }\end{array}$ & 0.2500 & 0.2500 & 91.7 \\
\hline
\end{tabular}

From Table 3, the best classification model derived from the established features, we used the GLCM method and obtained the highest accuracy of classification; the training model using 11 features of the model can be accurately, that only trained by 8 GLCMs to get the best gain the accuracy rate of classification. Although it can be noted that the accuracy of classification has reached better results, there is a great deal of contingency in the process of random data extraction, and the classification accuracy is very volatile. This shows that the construction of experimental model is not stable enough. The reason is the amount of data is too small and secondly, the feature extraction of images is not rich enough that resulted not enough in the available resolution feature representation.

\subsection{Experimental evaluation method}

For some features, such as gray and texture, the image of the same region should be consistent and uniform. Within the selected region it should be simple and have a low number of adjacent regions in the cavity, meet the features of regional internal consistency should have significant difference. The boundary of each region should be simple and rough, and the space position accurately. There are some evaluation indexes, including inter regional contrast, regional homogeneity, shape measurement, target counting consistency, pixel distance error, pixel number error, and the final measurement accuracy. Chang et al. introduced dice ratio, Hausdorff distance, Jaccard index, average perpendicular distance, conformity coefficient, precision, and recall [35]. Typically, the Dice ratio is a measure of set similarity which was widely used for evaluating performance of the segmenting results in image processing. Precision refers to the repeatability of segmentation applying to the same image data. In the current work, segmenting metrics of running time, dice ratio and precision were calculated, where the original source images shown in Fig. 12(a), and Fig. 12(b) is the standard image that was circled by experts.

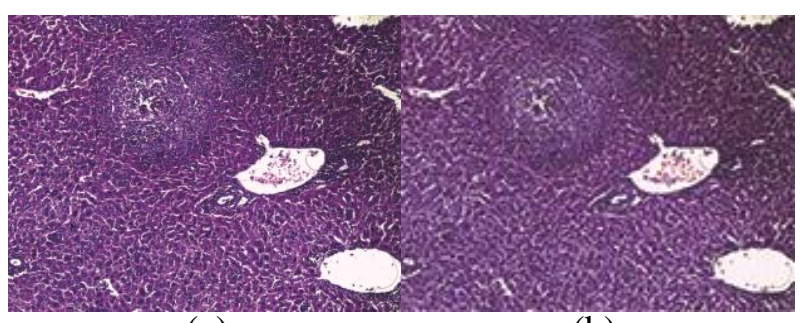

(a)

Figure 12 Source image and manual circled images 
The measured evaluation metrics are reported in Table 4, Fig. 13 and Table 5, namely the running time, dice ratio and precision; respectively. The comparative analysis on 16 fibrosis mice liver microscopic images was conducted using FCM, K-mean, Wd (watershed with distance transform), Wg (watershed with gradient) and Mt (morphological with local threshold), which is the proposed approach from this research.

Table 4. Comparing analysis of the running time in seconds of 16 fibrosis mice liver microscopic images using different algorithms

\begin{tabular}{|l|l|l|l|l|l|}
\hline Image & K-Means & FCM & $\mathrm{Wd}$ & $\mathrm{Wg}$ & Mt (Proposed) \\
\hline 1 & 15.469 & 32.908 & 3.322 & 8.556 & 2.507 \\
\hline 2 & 17.038 & 34.890 & 3.579 & 7.384 & 2.426 \\
\hline 3 & 17.827 & 30.109 & 3.437 & 7.464 & 2.457 \\
\hline 4 & 22.267 & 30.959 & 3.507 & 7.604 & 2.468 \\
\hline 5 & 17.460 & 34.081 & 2.990 & 7.537 & 2.412 \\
\hline 6 & 14.325 & 28.825 & 3.018 & 7.370 & 2.431 \\
\hline 7 & 17.444 & 38.551 & 4.035 & 8.913 & 2.454 \\
\hline 8 & 21.881 & 40.141 & 3.151 & 7.618 & 2.761 \\
\hline 9 & 13.555 & 36.206 & 2.964 & 7.469 & 2.065 \\
\hline 10 & 21.647 & 39.103 & 3.469 & 7.710 & 2.260 \\
\hline 11 & 20.025 & 38.721 & 2.943 & 7.843 & 2.419 \\
\hline 12 & 14.342 & 31.017 & 3.104 & 7.619 & 2.075 \\
\hline 13 & 21.243 & 27.893 & 3.557 & 7.611 & 2.124 \\
\hline 14 & 19.658 & 39.892 & 3.069 & 7.683 & 2.146 \\
\hline 15 & 21.880 & 33.296 & 3.056 & 7.627 & 2.205 \\
\hline 16 & 13.987 & 31.211 & 3.347 & 7.467 & 2.146 \\
\hline $\begin{array}{l}\text { Averag } \\
\text { e }\end{array}$ & 18.128 & 34.238 & 3.284 & 7.718 & 2.335 \\
\hline
\end{tabular}

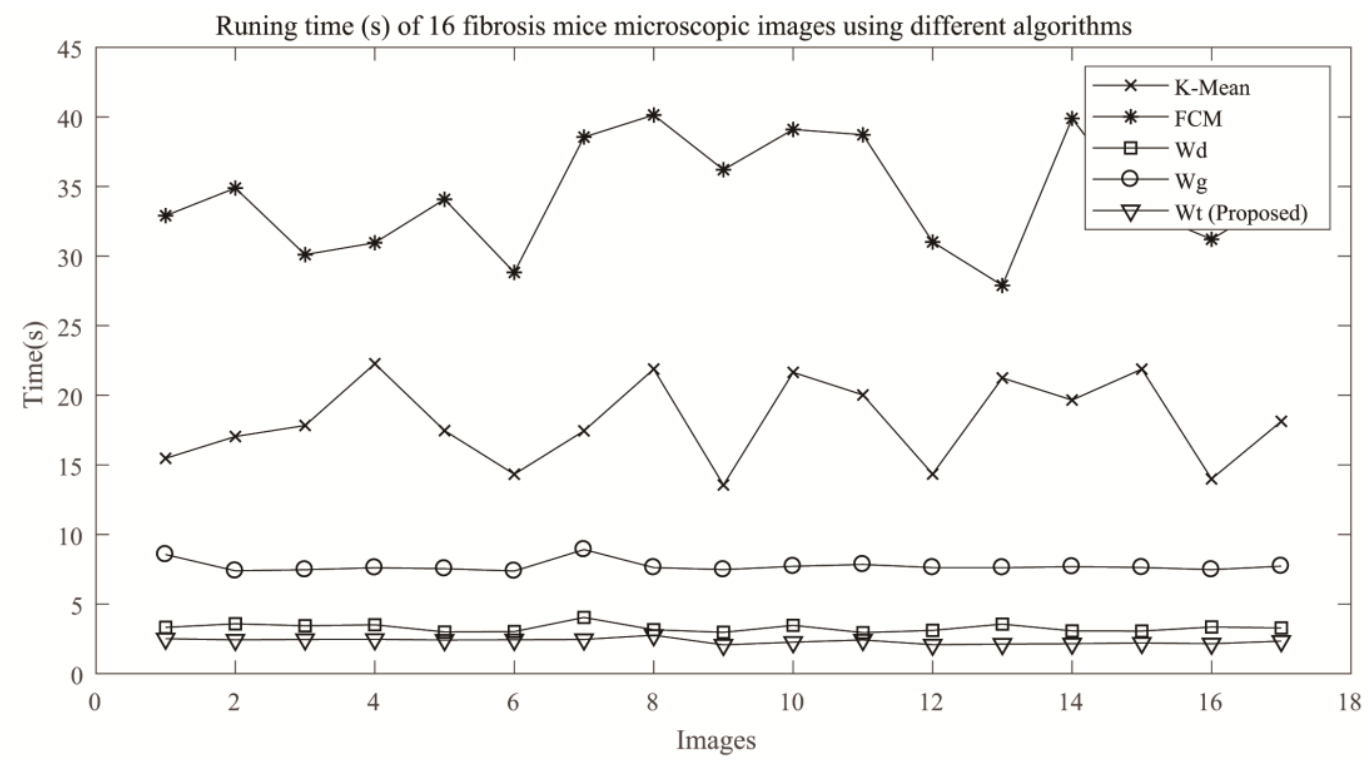

Figure 13. Comparison of the on running computation time of different segmenting algorithms

Table 5 Average segmenting metrics' values of dice ration and precision

\begin{tabular}{|l|l|l|}
\hline Method & Dice ratio & Precision \\
\hline FCM & 0.3214 & 0.1852 \\
\hline K-mean & 0.3475 & 0.1741 \\
\hline Wd & 0.5211 & 0.2144 \\
\hline Wg & 0.6102 & 0.2820 \\
\hline Mt (Proposed) & 0.8125 & 0.5253 \\
\hline
\end{tabular}


Table 5 reported that the proposed morphological with local threshold (Mt) algorithm performed the segmentation process within an average time of 2.335 seconds, which outperformed the other segmentation techniques. On the contrary, the FCM algorithm executed the segmentation process within an average time of 34.238 seconds, resulting in our algorithm being 14.6 times faster.

The preceding results depicted that the proposed local threshold based morphological segmentation has superior effective performance in processing fibrosis mice liver microscopic images. It took 2.335 seconds to perform the segmentation process with 0.8125 dice ratio and 0.5253 precision. Thus, it is recommended as a future work to use larger dataset to validate the processing algorithm proposed in this work that can also be evaluated using other metrics.

\section{CONCLUSIONS}

In the present work, the morphological segmentation, watershed segmentation, fuzzy c clustering and k-mean clustering were carried out to process microscopic images of mice liver fibrosis. The comparative experimental results reported that the proposed local threshold based morphological processing method from this research is feasible and effective in terms of evaluation indicators of running time, dice ratio and precision. Consequently, the present work makes the following contributions, including i) development of a local threshold based morphological segmenting method special for mice liver fibrosis microscopic images, ii) analyzed shortcomings of FCM, k-mean, watershed based algorithms on distance and gradient used in segmenting mice liver fibrosis microscopic images and iii) evaluated the segmenting results using indicators of running time, dice ratio and precision. The proposed algorithm is computationally efficient and was shown to process images 14.6 times faster than Fuzzy c-means, while also having superior dice ratio and precision performance.

\section{CONFLICT OF INTEREST}

We declare that we do not have any commercial or associative interest that represents a conflict of interest in connection with the work submitted.

\section{ACKNOWLEDGMENTS}

Dalia S. Ashour, Assistant professor of Medical Parasitology and Dina M. Aboraya, lecturer of Medical Parasitology, Medical Parasitology Department, Faculty of Medicine, Tanta University, Egypt performed the microscopic experiment and preprocessing the dataset. This work is supported by The National Natural Science Foundation of China (Grant nos: 81271663 and 31471146), Zhejiang Wenzhou Medical University Scientific Development Foundation of China (Grant no: QTJ06012) and Zhejiang Provincial Natural Science Foundation (Grant no: LY17F030014).

\section{REFERENCES}

[1] Jiang, W., \& Yin Z., Seeing the invisible in differential interference contrast microscopy images. Medical Image Analysis, 2016; 34, 65-81.

[2] Dey, N., Ashour, A.S., Ashour, A.S., \& Singh, A., Digital analysis of microscopic images in medicine. Journal of Advanced Microscopy Research, 2015; 10, 1-13.

[3] Dey, N., Ashour, A.S., Chakraborty, S., Samanta, S., Sifaki-Pistolla, D., Ashour, A.S., Le, D.-N., \& Nguyen, G.N., Healthy and unhealthy rat hippocampus cells classification: a neural based automated system for Alzheimer disease classification. Journal of Advanced Microscopy Research, 2016; 11, 1-10.

[4] Kotyk, T., Dey, N., Ashour, A.S., Drugarin, C.V.A., Gaber, T., Hassanien, A.E., Snasel. V. (2015, November). Detection of dead stained microscopic cells based on color intensity and contrast. Paper presented at the First International Conference on Advanced Intelligent Systems and Informatics, AISI2015, 28-30 Nov. 2015, Beni Suef, Egypt. Springer velarg, 2015.

[5] Rakotomamonjy, A., Petitjean, C., Salaün, M., \& Thiberville, L., Scattering features for lung cancer detection in fibered confocal fluorescence microscopy images. Artificial Intelligence in Medicine, 2014; 61, $105-118$.

[6] Tahir, M., \& Khan A., Protein subcellular localization of fluorescence microscopy images: Employing new statistical and Texton based image features and SVM based ensemble classification. Information Sciences, 2016; 345, 65-80.

[7] Nellros, F., Thurley, M. J., Jonsson, H., Andersson, C., \& Forsmo, S.P.E. (2015). Automated measurement of sintering degree in optical microscopy through image analysis of particle joins. Pattern Recognition, $2015 ; 48,3451-3465$.

[8] Ashour, A.S., Beagum, S., Dey, N., Ashour, A.S, Pistolla, D.S., Nguyen, G.N., Le, D.-N., \& Shi, F., Light microscopy image de-noising using optimized LPA-ICI filter. Neural Computing \& Applications. Online available: https://link.springer.com/article/10.1007\%2Fs00521-016-2678-9 
[9] Chun, M.G., \& Kong S.G., Focusing in thermal imagery using morphological gradient operator. Pattern Recognition Letters, 2014; 38, 20-25.

[10] Zia, S., Jaffar, M.A., \& Choi, T.S., Morphological gradient based adapted selective filter for removal of rician noise from magnetic resonance images. Microscopy Research and Technique, 2012; 75, 1044-1050.

[11] Li, B. Zhang, P. L., Mi, S.S., Hu, R.X., \& Liu, D.S., An adaptive morphological gradient lifting wavelet for detecting bearing defects. Mechanical Systems and Signal Processing, 2012; 29, 415-427.

[12] Khakipour, M.H., Safavi, A.A., \& Setoodeh, P., Bearing fault diagnosis with morphological gradient wavelet. Journal of the Franklin Institute, 2016; 354, 2465-2476.

[13] Dorini, F. A., Dorini, L.B., \& Lesinhovski, W.C., A mathematical analysis of the tensorial morphological gradient approach. Pattern Recognition Letters, 2015; 68, 97-102.

[15] Li, H., Li, L., \& Zhang, J., Multi-focus image fusion based on sparse feature matrix decomposition and morphological filtering. Optics Communications, 2015; 342, 1-11.

[16] Bai, X., Morphological center operator based infrared and visible image fusion through correlation coefficient. Infrared Physics \& Technology, 2016; 76, 546-554.

[17] Farihan, A., Raffei, M., Asmuni, H., Hassan, R., \& Othman, R.M., Frame detection using gradients fuzzy logic and morphological processing for distant color eye images in an intelligent iris recognition system. Applied Soft Computing, 2015; 37, 363-381.

[18] Gelzinis, A., Verikas, A., Vaiciukynas, E., Bacauskiene, M., Sulcius, S., Simoliunas, E., Staniulis, J., \& Paskauskas, R., Automatic detection and morphological delineation of bacteriophages in electron microscopy images. Computers in Biology and Medicine, 2015; 64, 101-116.

[19] Preziosi, B.M., \& Bowden, T.J., Morphological characterization via light and electron microscopy of Atlantic jackknife clam (Ensis directus) hemocytes. Micron, 2016; 84, 96-106.

[20] Oschatz, M., Pré, P., Dörfler, S., Nickel, W., Beaunier, P., Rouzaud, J.N., Fischer, C., Brunner, E., \& Kaskel, S., Nanostructure characterization of carbide-derived carbons by morphological analysis of transmission electron microscopy images combined with physisorption and Raman spectroscopy. Carbon, 2016; 105, 314-322.

[21] Kayasandik, C.B., \& Labate, D., Improved detection of soma location and morphology in fluorescence microscopy images of neurons. Journal of Neuroscience Methods, 2016; 274, 61-70.

[22] Yamamoto, S., Oshima, Y., Saitou, T., Watanabe, T., Miyake, T., Yoshida, O., Yoshio, T., Abe, M., Matsuura, B., Hiasa, Y., \& Imamura T., Quantitative imaging of fibrotic and morphological changes in liver of non-alcoholic steatohepatitis (NASH) model mice by second harmonic generation (SHG) and auto-fluorescence (AF) imaging using two-photon excitation microscopy (TPEM), Biochemistry and Biophysics Reports, 2016; 8, 277-283.

[23] López-Mir, F., Naranjo, V., Angulo, J., Alcañiz, M., \& Luna, L., Liver segmentation in MRI: A fully automatic method based on stochastic partitions. Computer Methods and Programs in Biomedicine, 2014; $114,11-28$.

[24] Das, A., \& Ghoshal, D., Human skin region segmentation based on chrominance component using modified watershed algorithm. Procedia Computer Science, 2016; 89, 856-863.

[25] Wong, A.K.O., Hummel, K., Moore, C., Beattie, K.A., Shaker, S., Craven, B.C., Adachi, J.D., Papaioannou, A., \& Giangregorio, L., Improving reliability of pQCT-derived muscle area and density measures using a watershed algorithm for muscle and fat segmentation. Journal of Clinical Densitometry, $2015 ; 18,93-101$.

[26] Masoumi, H., Behrad, A., Pourmina, M.A., \& Roosta, A., Automatic liver segmentation in MRI images using an iterative watershed algorithm and artificial neural network. Biomedical Signal Processing and Control, 2012; 7, 429-437.

[27] Wieclawek, W., \& Pietka E., Watershed based intelligent scissors. Computerized Medical Imaging and Graphics, 2015; 43, 122-129.

[28] Gatiatulina, E.R., Popova, E.V., Polyakova, V.S., Skalnaya, A.A., Agletdinov, E.F., Nikonorov, A.A., Skalny, A.V., \& Tinkov, A. A., Evaluation of tissue metal and trace element content in a rat model of non-alcoholic fatty liver disease using ICP-DRC-MS. Journal of Trace Elements in Medicine and Biology, 2017; 39, 91-99.

[29] Hore, S., Chakroborty, S., Ashour, A.S., Dey, N., Ashour, A.S., Sifaki-Pistolla, D., \& Chaudhuri, S.R., Finding contours of hippocampus brain cell using microscopic image analysis. Journal of Advanced Microscopy Research, 2015; 10, 93-103.

[30] Li, C.H., Ge, X.L., Pan, K., Wang, P.F., Su, Y.N., \& Zhang, A.Q., Laser speckle contrast imaging and Oxygen to See for assessing microcirculatory liver blood flow changes following different volumes of hepatectomy. Microvascular Research, 2017; 110, 14-23.

[31] Vreuls, C.P.H., Driessen, A., Damink, S.W.M.O., Koek, G.H., Duime, H. 1., van den Broek, M.A.J., Dejong, C.H.C., Braet, F., \& Wisse, E., Sinusoidal obstruction syndrome (SOS): A light and electron microscopy study in human liver. Micron, 2016; 84, 17-22. 
[32] Sayed, G.I., Hassanien, A.E., \& Schaefer, G., An automated computer-aided diagnosis system for abdominal CT liver images. Procedia Computer Science, 2016; 90, 60-73.

[33] Li W., Design and implementation of CAD system based on multiphase liver images, PhD Thesis, Shanghai Jiaotong University, 2010

[34] Theodoridis, S., \& Koutroumbas, K., Pattern Recognition. Beijing, China: Publishing House of Electronics Industry, 2000.

[35] Chang, H.H., Zhuang, A.H., Valentino, D.J., \& Chua, W.C., Performance measure characterization for evaluating neuroimage segmentation algorithms. Neuroimage, 2009; 47, 122-135. 